\title{
Vegetable-derived isothiocyanates: anti-proliferative activity and mechanism of action
}

\author{
Yuesheng Zhang*, Song Yao and Jun Li \\ Department of Chemoprevention, Roswell Park Cancer Institute, Buffalo, New York 14263, USA
}

\begin{abstract}
Many isothiocyanates (ITC), which are available to human subjects mainly through consumption of cruciferous vegetables, demonstrate strong cancer-preventive activity in animal models. Human studies also show an inverse association between consumption of ITC and risk of cancer in several organs. Whereas earlier studies primarily focused on the ability of ITC to inhibit carcinogen-activating enzymes and induce carcinogen-detoxifying enzymes, more recent investigations have shown that ITC inhibit the proliferation of tumour cells both in vitro and in vivo by inducing apoptosis and arresting cell cycle progression. ITC cause acute cellular stress, which may be the initiating event for these effects. These findings shed new light on the mechanism of action of ITC and indicate that ITC may be useful both as cancer-preventive and therapeutic agents. ITC activate caspase 9-mediated apoptosis, apparently resulting from mitochondrial damage, and also activate caspase 8 , but the mechanism remains to be defined. Cell cycle arrest caused by ITC occurs mainly in the $G_{2} / M$ phase, and both the $G 2$ and $\mathrm{M}$ phases are targetted; critical $\mathrm{G}_{2}$-phase regulators, including cyclin $\mathrm{B} 1$, cell division cycle (Cdc) 2 and $\mathrm{Cdc} 25 \mathrm{C}$, are down regulated or inhibited, and tubulin polymerization and spindle assembly are disrupted. Moreover, ITC are metabolized in vivo through the mercapturic acid pathway, giving rise to thiol conjugates (dithiocarbamates). Studies show that these dithiocarbamates are similar to their parent ITC in exerting anti-proliferative activity. Taken together, dietary ITC are highly-promising anti-cancer agents, capable of targetting multiple cellular components that are important for tumour cell survival and proliferation.
\end{abstract}

Isothiocyanate: Anti-cancer agent: Cancer chemopreventive agent

All isothiocyanates (ITC) are characterized by the presence of a $-\mathrm{N}=\mathrm{C}=\mathrm{S}$ group, and many lines of evidence indicate that the biological activities of ITC may be primarily mediated through the reaction of the electrophilic central $\mathrm{C}$ of $-\mathrm{N}=\mathrm{C}=\mathrm{S}$ with cellular nucleophilic targets. Since $\alpha$-napthyl ITC was first shown to inhibit liver tumourigenesis induced by chemical carcinogens in rats about 40 years ago (Sasaki, 1963; Sidransky et al. 1966), numerous studies have not only confirmed these early findings but have also uncovered the cancer-preventive activities of other ITC (Zhang \& Talalay, 1994; Hecht, 2000). Of particular importance is the finding that many of the ITC that show strong tumour-inhibitory effects in rodents are present in commonly-consumed cruciferous vegetables, such as broccoli, cabbage and watercress (Nasturtium officinale), and are therefore widely consumed by human subjects. Indeed, several recent epidemiological studies have reported an inverse association between consumption of dietary ITC and cancer risk in several organs (London et al. 2000; Spitz et al. 2000; Zhao et al. 2001; Seow et al. 2002; Fowke et al. 2003). These findings have provided an explanation for the widely-recognized cancer-preventive activity of cruciferous vegetables, i.e. ITC may be part of the active ingredients. Interestingly, available evidence suggests that ITC are probably absent in normal or fresh plants (Fahey et al. 1997) but are rapidly generated from $\beta$-thioglucoside $N$-hydroxysulfates (glucosinolates) through a hydrolysis reaction catalysed by the coexisting myrosinase (thioglucoside glucohydrolase) on damage of plant cells (Fenwick et al. 1983). Many cruciferous vegetables are abundant in certain glucosinolates, but the compounds are segregated from myrosinase in normal plant cells.

Although early studies mainly focused on the ability of ITC to inhibit the formation of cancer cells, i.e. inhibition of carcinogenesis, it is now well-known that ITC can also

\footnotetext{
Abbreviations: AITC, allyl isothiocyanate; BITC, benzyl isothiocyanate; Cdc, cell division cycle; GSH, glutathione; ITC, isothiocyanate; NAC, N-acetylcysteine; PEITC, phenylethyl isothiocyanate, ROS, reactive oxygen species; SF, sulforaphane.

*Corresponding author: Dr Y. Zhang, fax + 1716845 1144, email yuesheng.zhang@ roswellpark.org
} 
suppress the survival and proliferation of existing cancer cells (the anti-cancer activity). The molecular basis for both the anti-carcinogenic and anti-cancer effects of ITC has been extensively investigated, showing that ITC can target cancer in multiple directions, including inhibition of carcinogen-activating enzymes, induction of carcinogendetoxifying enzymes, induction of apoptosis and arrest of cell cycle progression, as well as other mechanisms that are not yet well understood (Conaway et al. 2002; Kaum et al. 2004; Zhang, 2004; Zhang et al. 2005). Among these mechanisms, however, induction of apoptosis and cell cycle arrest are of particular interest as they may be exploited for both cancer prevention and cancer treatment. The present review will examine current knowledge about the impact of dietary ITC on the survival and proliferation of cancer cells and their modulation of apoptosis and cell cycle progression.

\section{Anti-proliferative effects of dietary isothiocyanates}

Numerous studies have reported the inhibitory effects of ITC on the growth of both animal and human cancer cells in culture. Overall, these studies show that ITC inhibit cell growth at low micromolar concentrations and their activities are not cell specific. Moreover, ITC may selectively target transformed or malignant cells, as they are less potent against the growth of normal cells (Musk \& Johnson, 1993; Gamet-Payrastre et al. 1998; Srivastava et al. 2003; Choi \& Singh, 2005). There are other antiproliferative properties of ITC that are also highly interesting. First, exposure of cells to ITC, including allyl ITC (AITC), benzyl ITC (BITC) and phenylethyl ITC (PEITC) for only $3 \mathrm{~h}$ is long enough to inhibit cell growth, indicating a rapid and irreversible interaction of these ITC with cellular targets (Zhang et al. 2003; Tang \& Zhang, 2004). This finding is important because it suggests that the anti-proliferative activity of the compounds may not be markedly affected by their rapid disposal kinetics in vivo (Ioannou et al. 1984; Ye et al. 2002; Ji \& Morris, 2003). Second, drug-resistant cancer cells that overexpress multidrug-resistance-associated protein-1 and P-glycoprotein-1 are sensitive to ITC (Zhang et al. 2003). In this context it is noteworthy that Morris and co-workers (Hu \& Morris, 2004; Ji \& Morris, 2004) have recently reported that ITC are inhibitors of multidrug-resistanceassociated protein-1, P-glycoprotein-1 and other drug transporters. Third, metabolic conversion of ITC in vivo may not markedly affect the anti-proliferative activity of these compounds. ITC are metabolized in vivo primarily through the mercapturic acid pathway, where an initial conjugation of ITC with glutathione (GSH) gives rise to the corresponding GSH conjugates, which then undergo further enzymic modifications to form sequentially the cysteinylglycine, cysteine and $N$-acetylcysteine (NAC) conjugates, which are excreted in the urine (Brusewitz et al. 1977). It has been shown (Xu \& Thornalley, 2000; Zhang et al. 2003) that the conjugates display potencies very similar to their parent ITC in inhibiting the growth of cancer cells. These conjugates (generally termed dithiocarbamates) are considered to be the carriers of ITC, because they are unstable and readily dissociate to the parent ITC (Conaway et al. 2001).

Several ITC have also been shown to inhibit the growth of cancer cells in vivo. Singh and co-workers (Srivastava et al. 2003; Singh AV et al. 2004; Xiao et al. 2005) have reported that the growth of mouse or human prostate cancer cells that are subcutaneously inoculated in mice is retarded by AITC $(10 \mu \mathrm{mol}$ per mouse by intraperitoneal injection, three times per week), PEITC (9-12 $\mu$ mol per mouse by oral intubation three times per week) or sulforaphane (SF; $5.6 \mu \mathrm{mol} / \mathrm{kg}$ diet daily). Moreover, Chiao et al. (2004) have also reported that oral administration of the NAC conjugate of PEITC $(8 \mu \mathrm{mol} / \mathrm{g}$ diet daily) inhibits the proliferation of human prostate cancer cell xenograft in mice. Tumours harvested from mice treated with the ITC or the PEITC metabolite show a decrease in cell proliferation and an increase in apoptosis. These results clearly show the in vivo anti-cancer activity of dietary ITC and suggest that they are potentially useful for treating prostate cancers in man.

\section{Isothiocyanates cause acute cellular stress}

ITC are dichotomous modulators of cellular stress and cell survival; exposure of cells to ITC rapidly leads to an increase in stress and perhaps cell death but elicits a delayed induction of antioxidative or anti-carcinogenic enzymes and increased detoxification capacity (Zhang et al. 2005). While the induction of cellular protective enzymes and detoxification capacity by ITC will not be reviewed here, as it does not directly relate to the anti-proliferative activities of the compounds and much information is available elsewhere (Zhang, 2004; Kaum et al. 2004; Zhang et al. 2005), understanding how ITC cause cellular stress may be key to the elucidation of the mechanism responsible for their anti-proliferative effects.

\section{Isothiocyanates cause alkylation and depletion of cellular glutathione}

Most ITC are electrophiles because of the presence of a $-\mathrm{N}=\mathrm{C}=\mathrm{S}$ group, which can react with various nucleophiles, especially those that are S-based (thiocarbamoylation). Indeed, the strong reaction of ITC with cellular thiols, particularly GSH (the most abundant intracellular thiol), is primarily responsible for the rapid accumulation of ITC in cells, which has recently been observed in the authors' laboratory (Fig. 1). For example, when murine hepatoma Hepa1c1c7 cells are exposed to SF or BITC at $50 \mu \mathrm{M}$ for $30 \mathrm{~min}$ the total intracellular ITC accumulations are $5 \cdot 1$ and $5 \cdot 9 \mathrm{~mm}$ respectively, a $110-190$-fold increase over the extracellular ITC concentration, but $98 \% \mathrm{SF}$ and $62 \%$ BITC are present in the cell as GSH conjugates (Zhang, 2000). ITC appear to penetrate a cell by diffusion, but once in the cell they are rapidly conjugated with GSH and other thiols (Zhang, 2001). Not surprisingly, under the conditions described intracellular GSH concentration drops from $7.3 \mathrm{~mm}$ to $3.3-3.7 \mathrm{~mm}$ (49-55\% depletion; Zhang, 2000). Similar effects of these and other ITC on GSH have been reported in other cells (Xu \& Thornalley, 2001a; Kim 


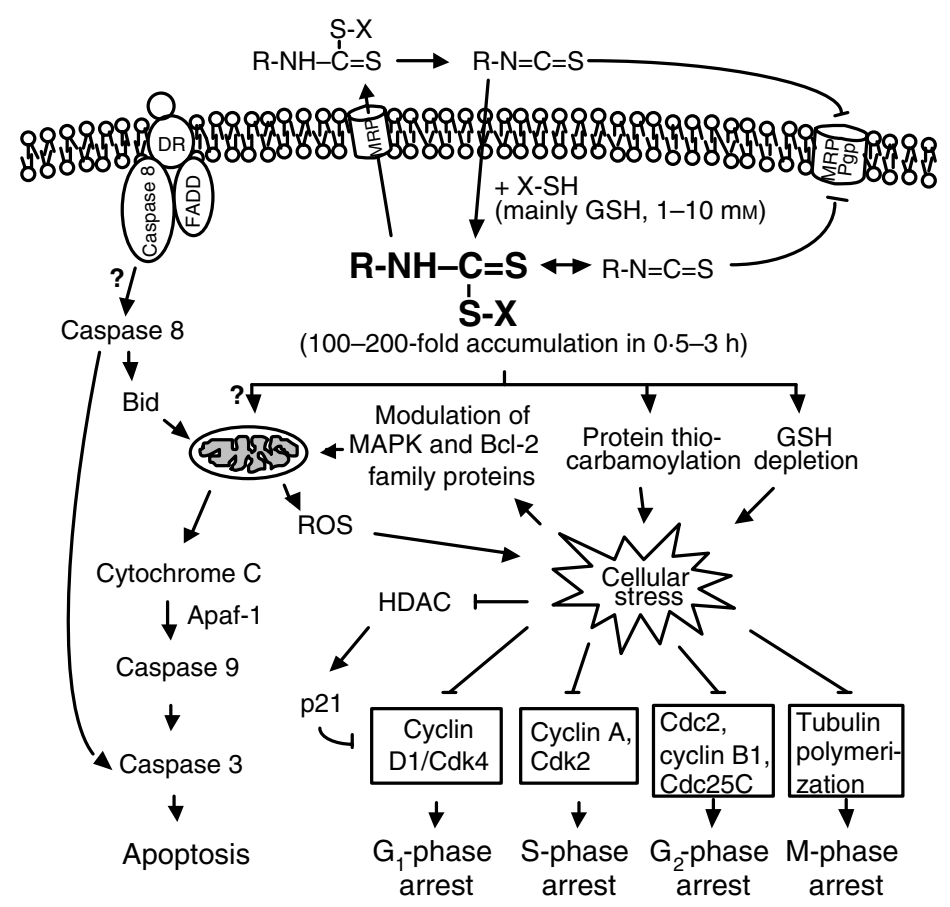

Fig. 1. A schematic representation of the known or suggested signalling pathways in isothiocyanate-induced apoptosis and cell cycle arrest. $\mathrm{R}-\mathrm{N}=\mathrm{C}=\mathrm{S}$, isothiocyanate; $\mathrm{DR}$, death receptor; FADD, Fas-associated protein with death domain; $\mathrm{GSH}$, glutathione; $\mathrm{X}$, thiol compounds, such as GSH; Apaf-1, apoptosis protease activation factor-1; MRP, multidrugresistance-associated protein; ROS, reactive oxygen species; MAPK, mitogen-activated protein kinase; HDAC, histone deacetylase; Cdk, cyclindependent kinase; Cdc, cell division cycle; Pgp, P-glycoprotein.

et al. 2003; Hudson et al. 2005). Moreover, because ITC conjugated with GSH are rapidly removed by membrane efflux pumps (Zhang \& Callaway, 2002; Callaway et al. 2004), and the expelled conjugates may release ITC for re-accumulation in cells (Zhang \& Callaway, 2002), the depletion of GSH by ITC may be especially marked and efficient (Fig. 1). Depletion of GSH undoubtedly renders cells more susceptible to stress and stress-induced injury and may trigger apoptosis (Hall, 1999). However, the rapid and dramatic alkylation and depletion of GSH by ITC is largely transient and contrasts with a delayed but prolonged increase in GSH levels in ITC-treated cells (Zhang \& Talalay, 1998; Ye \& Zhang, 2001; Xu \& Thornalley, 2001a; Kim et al. 2003; Tang \& Zhang, 2004), apparently resulting, at least in part, from increased GSH biosynthesis in response to induction of glutamate cysteine ligase (Zhang et al. 2002).

\section{Isothiocyanates damage mitochondria and generate reactive oxygen species in cells}

Mitochondrial damage caused by ITC is both rapid and profound. Mitochondrial damage is evident after human hepatoma HepG2 cells are exposed to $20 \mu \mathrm{M}$-PEITC for 15 min or rat liver epithelial RL34 cells are exposed to $5 \mu \mathrm{M}$-BITC for $30 \mathrm{~min}$, as shown by a loss of mitochondrial trans-membrane potential (Nakamura et al. 2002; Rose et al. 2005). Incubation of human leukaemia HL60 cells with AITC and BITC at $10 \mu \mathrm{M}$ for $3 \mathrm{~h}$ results in mitochondrial trans-membrane potential loss in 44 and $77 \%$ of the cells respectively, as compared with $13 \%$ of the cells in the control (Zhang et al. 2003). Studies indicate that ITC-induced mitochondrial damage is not cell specific (Nakamura et al. 2000, 2002; Payen et al. 2001; Rose et al. 2003; Tang \& Zhang, 2005; Xiao et al. 2005). Cell exposure to ITC apparently causes damage to both outer and inner mitochondrial membranes, as both cytochrome c (normally residing in the inter-membrane space) and malate dehydrogenase (normally residing in the mitochondrial matrix) are detected in the cytosolic fraction after ITC treatment (Nakamura et al. 2002; Tang \& Zhang, 2005). Moreover, treatment of isolated mitochondria with GSH conjugates of ITC (the principal ITC metabolites in cells) leads to mitochondrial damage, indicating that these conjugates may be the primary cause of mitochondrial damage in ITC-treated cells (Tang \& Zhang, 2005). The exact mechanism by which ITC or their GSH conjugates damage mitochondria remains largely unknown. Several synthetic ITC, including $p$-bromophenyl ITC, 4,4'-diisothiocyanatebiphenyl and $\beta$-naphthylmethyl ITC, have been shown to uncouple oxidative phosphorylation (Miko \& Chance, 1975), but it is not known if any dietary ITC have the same effect. Bcl-2 family proteins are known to either stabilize (Bcl-2, Bcl-xl and Mcl-1) or destabilize 
(Bad, Bak, Bax and Bid) the mitochondrial membrane. Singh and co-workers (Choi \& Singh, 2005; Xiao et al. 2005) have recently shown that cells deficient in Bak and/or Bax are resistant to death induced by SF and PEITC. The known effects of ITC on Bcl-2 family proteins include: inactivation of $\mathrm{Bcl}-2$ via its phosphorylation by c-Jun N-terminal kinase; down-regulation of Bcl-2, Bcl-xl and Mcl-1; activation of Bid; up-regulation of Bad, Bak and Bax; increased mitochondrial translocation of Bax (Xu \& Thornalley, 2001b; Xiao et al. 2003; Miyoshi et al. 2004; Srivastava \& Singh, 2004; Singh SV et al. 2004; Xiao et al. 2004; Choi \& Singh, 2005; Rose et al. 2005; Tang \& Zhang, 2005; Xiao et al. 2005). Moreover, it has recently been found (Tang \& Zhang, 2005) that treatment of mitochondria isolated from human bladder cancer UM-UC-3 cells with BITC disrupts the association of Bcl-xl with Bak and Bax. However, available information suggests that these effects are somewhat cell specific. For example, treatment of HepG2 cells with $20 \mu \mathrm{M}$-PEITC causes the level of cytosolic Bax to increase, as well as translocation of Bax to the mitochondria (Rose et al. 2005), whereas treatment of murine prostate cancer cells (TRAMP-C1 and TRAMP-C2) with $10 \mu \mathrm{M}-\mathrm{PEITC}$ either does not affect (TRAMP-C1) or actually reduces (TRAMP-C2) the Bax protein level and does not affect Bax mitochondrial translocation in either cell line (Xiao et al. 2005). Mitogen-activated protein kinases may also play a role in ITC-induced mitochondrial damage, as described later.

Since reactive oxygen species (ROS) are produced in the mitochondria and may leak out of damaged mitochondria, it is not surprising that many studies have shown an increase in intracellular ROS levels in ITC-treated cells. Indeed, ROS generation seems to correspond with mitochondrial damage in ITC-treated cells. For example, mitochondrial damage and ROS generation are detected after incubation of rat liver RL34 cells with 5-10 $\mu \mathrm{M}$-BITC for $30 \mathrm{~min}$ (Nakamura et al. 2000). Also, while an increase in both superoxide and $\mathrm{H}_{2} \mathrm{O}_{2}$ are detected in ITC-treated cells, $\mathrm{H}_{2} \mathrm{O}_{2}$ seems to be derived from dismutation of superoxide, which mainly occurs in mitochondria (Nakamura et al. 2002). Moreover, similar to mitochondrial damage caused by ITC, ROS generation by ITC is neither cell nor ITC specific (Payen et al. 2001; Rose et al. 2003; Xiao et al. 2005). While their excessive production probably causes non-specific cytotoxicity, ROS appear to play an important role in apoptosis (Buttke \& Sandstrom, 1994) and cell cycle arrest (Shackelford et al. 2000). In fact, SF-induced cell death in human prostate cancer cells is initiated by increased ROS production (Singh et al. 2005), and PEITC-induced apoptosis in human leukaemia Jurkat T cells and HL60 cells is inhibited by antioxidants, including NAC and GSH (Chen et al. 1998; Xu \& Thornalley, 2001a).

\section{Isothiocyanates induce apoptosis}

Induction of apoptosis by ITC was first reported by Tan and co-workers (Chen et al. 1998), who showed that BITC and PEITC at $5 \mu \mathrm{M}$ induces apoptosis in Jurkat cells through a c-Jun N-terminal kinase-mediated mechanism.
Since then, numerous studies have shown the apoptosisinducing activities of a large number of ITC in a variety of cancer cell lines in culture and tumour xenografts in mice in vivo. ITC-treated cells frequently show activation of two initiator caspases, caspase 8 and caspase 9, indicative of activation of both death receptor signalling and mitochondria signalling (Fig. 1), although it is often difficult to know which signalling pathway may play a more important role in ITC-induced apoptosis.

The majority of studies, however, have focused on the mitochondria-mediated apoptosis pathway. Mitochondriamediated apoptosis is initiated when cytochrome $\mathrm{c}$ is released from the mitochondrial inter-membrane space into the cytoplasm, where together with apoptosis protease activation factor-1 it recruits and activates caspase 9. Activated caspase 9 in turn activates effector caspases, including caspase 3 , leading to apoptotic cell death. In line with the finding that treatment of cells with ITC causes mitochondrial damage, as described earlier, many studies (for example, see Nakamura et al. 2002; Hu et al. 2003; Zhang et al. 2003; Rose et al. 2005; Tang \& Zhang, 2005; Xiao et al. 2005) have reported the release of cytochrome c from mitochondria to the cytoplasm as well as activation of caspase 9 and caspase 3 in ITC-treated cells. Moreover, studies in both human leukaemia HL60 cells and UMUC-3 cells (Zhang et al. 2003; Tang \& Zhang, 2005), as well as in murine embryonic fibroblasts (Choi \& Singh, 2005), have shown that mitochondrial release of cytochrome c and activation of caspase 9 and caspase 3 occur as early as $1-3 \mathrm{~h}$ after cell exposure begins, coinciding with the time when mitochondria are damaged by ITC.

Current knowledge about the role of $\mathrm{Bcl}-2$ family proteins in mediating ITC-induced apoptosis is incomplete. Nevertheless, a number of Bcl-2 family proteins that have been shown to be modulated by ITC (as described earlier) and may play a role in ITC-induced apoptosis, including Bcl-2, Bcl-xl, Bad, Bak, Bax and Bid, probably exert their effects through mitochondria (Chen et al. 1998; Xu \& Thornalley, 2001b; Hu et al. 2003; Choi \& Singh, 2005; Xiao et al. 2005). The three mitogen-activated protein kinases, including c-Jun N-terminal kinase, extracellular signal-regulated protein kinases and p38 kinases, have also been shown to be rapidly activated by ITC and contribute to apoptosis in a somewhat cell-specific fashion (Xiao \& Singh, 2002; Hu et al. 2003; Lui et al. 2003; Miyoshi et al. 2004). Information on exactly how the mitogen-activated protein kinases mediate ITC-induced apoptosis is unavailable, but it seems likely that mitochondria are involved in their actions: c-Jun N-terminal kinase is known to inactivate Bcl-2 (Li et al. 2005); both extracellular signalregulated protein kinases and p38 can phosphorylate p53, which in turn modulates Bax (Miyashita \& Reed, 1995); extracellular signal-regulated protein kinases may also modulate Bad (Scheid et al. 1999). The role of p53 in ITC-induced apoptosis, however, is also cell specific, as it has been reported to be essential in PEITC-induced apoptosis in JB6 mouse epidermal cells (Huang et al. 1998), but PEITC potently induces apoptosis in both p53-deficient PC-3 human prostate cancer cells (Xiao \& Singh, 2002) and p53-deficient HL60 cells (Xu \& Thornalley, 2000). 
ITC may also modulate other factors involved in the mitochondria-mediated apoptosis. Recently, it has been shown (Choi \& Singh, 2005) that when murine embryonic fibroblasts are incubated with SF for 8-24h: Smac/DIABLO, which is known to counter caspase inhibitors, is activated (released from mitochondria to cytoplasm); apoptosis protease activation factor-1, which facilitates caspase 9 activation by cytochrome c, is up regulated; $\mathrm{X}$-linked inhibitor of apoptosis, which inhibits caspases, is down regulated. However, whether these changes also occur in other cells, or with other ITC, remains to be investigated.

Caspase 8 is another initiator caspase that is frequently activated by ITC (Xu \& Thornalley, 2000; Zhang et al. 2003; Pham et al. 2004; Tang \& Zhang, 2004, 2005; Xiao et al. 2004). Activated caspase 8 can directly activate effector caspases such as caspase 3 (Thornberry \& Lazebnik, 1998), or cleave (activate) Bid, which then interacts with Bcl-xl and causes mitochondrial release of cytochrome c (Li et al. 1998). Indeed, Xu \& Thornalley $(2001 b)$ have shown that treatment of HL60 cells with $10 \mu \mathrm{M}$-PEITC for $6 \mathrm{~h}$ results in activation of Bid. However, how ITC activate caspase 8 is presently unknown, although caspase 8 is known to be downstream of death receptor signalling (Ashkenazi \& Dixit, 1998). Interestingly, treatment of a number of cell lines with either AITC or SF has recently been shown to result in increased histone acetylation, apparently a result of inhibition of histone deacetylase (Lea et al. 2001; Myzak et al. 2004). Non-ITC histone deacetylase inhibitors have been shown to induce the expression of death receptor signalling (both death receptor and their ligands are up regulated) in leukaemia cells by increasing promoter region histone acetylation (Insinga et al. 2005; Nebbioso et al. 2005). Whether ITC activate caspase 8 through inhibition of histone deacetylase remains to be investigated. However, Singh et al. (2005) have recently reported that SF treatment of PC-3 cells leads to an increase in Fas level (one of the death receptor family members), accompanied by activation of caspase 8 and Bid.

\section{Isothiocyanates arrest cell cycle progression}

The ability of an ITC to arrest cell cycle progression was first reported by Hasegawa et al. (1993), who showed that HeLa cells are arrested in the $\mathrm{G}_{2} / \mathrm{M}$ phase after incubation with AITC, BITC or PEITC at $2 \cdot 5-10 \mu \mathrm{m}$ for $16 \mathrm{~h}$. Since then, these and other ITC, as well as particular metabolites of ITC (NAC conjugates), have been repeatedly shown to arrest cell cycle progression in a wide variety of cell lines. Whereas the majority of cell lines tested are arrested by ITC in the $G_{2} / M$ phase, in a few cell lines the same ITC cause $\mathrm{G}_{1}$-phase or S-phase arrest (Chiao et al. 2000; Zhang et al. 2003; Tang \& Zhang, 2004). Little is known about the differential cell cycle arrest effects among cell lines. In this context, however, it is of interest to note that both human prostate cancer LNCaP cells (androgendependent and p53-intact) and DU-145 cells (androgenindependent and p53-mutated) are arrested by the NAC conjugate of PEITC in the $\mathrm{G}_{1}$ phase (Chiao et al.
2000), whereas PC-3 cells (androgen-independent and p53-mutated) are arrested by PEITC in the $G_{2} / M$ phase (Xiao et al. 2004). The differential effects are probably not a result of different behaviours of PEITC and NACPEITC, as NAC conjugates of ITC are known to exert their effects by releasing ITC. Indeed, both SF and its NAC conjugate block LNCaP cells in the $\mathrm{G}_{1}$ phase (Chiao et al . 2002), but SF blocks PC-3 cells in the $\mathrm{G}_{2} / \mathrm{M}$ phase (Singh SV et al. 2004).

$\mathrm{G}_{1}$-phase arrest of LNCaP and DU-145 cells treated with SF, NAC-SF or NAC-PEITC is associated with either increased p21 expression or decreased cyclin D1 expression (Chiao et al. 2000, 2002). p21 is a negative $\mathrm{G}_{1}$-phase regulator and cyclin $\mathrm{D} 1$ is a positive $\mathrm{G}_{1}$-phase regulator, suggesting that these proteins may mediate $\mathrm{G}_{1}$-phase arrest by $\mathrm{SF}$ and the NAC-ITC conjugates (Fig. 1). Treatment of UM-UC-3 cells with AITC, BITC or PEITC causes S-phase arrest, in addition to $\mathrm{G}_{2} / \mathrm{M}$-phase arrest (Tang \& Zhang, 2004), and the expression levels of cyclin A and cyclin-dependent kinase 2 (the primary S-phase regulators) appeared to be reduced (Fig. 1).

Although in the majority of published papers the impact of ITC on the $G_{2}$ and $M$ phases have been described indiscriminatingly, it is now increasingly evident that ITC arrest cells in both the $\mathrm{G} 2$ and $\mathrm{M}$ phases by targetting corresponding regulators. The cyclin B-cell division cycle (Cdc) 2 complex plays a critical role in cell transition from the $G_{2}$ phase to the $M$ phase (Shackelford et al. 2000), but it requires activation by $\mathrm{Cdc} 25 \mathrm{C}$, a phosphatase that removes the inhibitory phosphates on $\mathrm{T}-14 / \mathrm{Y}-15$ of $\mathrm{Cdc} 2$ (Shackelford et al. 2000). Published data have shown that ITC can target all three proteins (Fig. 1). For example, PEITC in HepG2 cells down regulates Cdc2 (Rose et al. 2003) and BITC and PEITC down regulate both cyclin B1 and Cdc2 in UM-UC-3 cells (Tang \& Zhang, 2004). Singh and co-workers (Xiao et al. 2003, 2004; Singh SV et al. 2004) have shown that ITC, including AITC, PEITC and $\mathrm{SF}$, down regulate $\mathrm{Cdc} 25 \mathrm{C}$ in $\mathrm{PC}-3$ cells, in addition to down-regulation of cyclin $\mathrm{B} 1$ and $\mathrm{Cdc} 2$ (AITC and PEITC) or cyclin B1 (SF). The level of $\mathrm{Cdc} 25 \mathrm{~B}$, the function of which may be similar to that of $\mathrm{Cdc} 25 \mathrm{C}$, is also reduced in AITC- and SF-treated PC-3 cells. While it is not clear how the ITC down regulate cyclin $\mathrm{B} 1$ and $\mathrm{Cdc} 2$, ITC-induced down-regulation of $\mathrm{Cdc} 25 \mathrm{C}$ appears to result, at least in part, from its phosphorylation at Ser-216 by checkpoint kinase 2 and translocation of phosphorylated Cdc $25 \mathrm{C}$ from the nucleus to the cytoplasm as a result of increased binding with 14-3-3 protein and subsequent proteasomemediated degradation (Singh SV et al. 2004; Xiao et al. 2004).

Both SF and AITC have been shown to arrest cells in the $\mathrm{M}$ phase, in addition to causing $\mathrm{G}_{2}$-phase arrest SF-induced $\mathrm{M}$-phase arrest is observed in murine mammary cancer F3II cells, human breast cancer MCF-7 cells and human pancreatic cancer MIA PaCa-2 cells and PANC-1 cells (Jackson \& Singletary 2004a,b; Pham et al. 2004). AITC has a similar effect on human colon cancer HT-29 cells (Smith et al. 2004). These results therefore suggest that $\mathrm{M}$-phase arrest is neither cell nor ITC specific. Both AITC and SF apparently target tubulin, disrupting intracellular tubulin 
polymerization and spindle assembly, thereby inhibiting mitosis (Fig. 1).

\section{Summary}

Dietary ITC demonstrate potent anti-proliferative activity in cultured cell lines and animal models and their ability to attack multiple survival-related targets and signalling pathways. However, further study is needed to fully understand the anti-proliferative mechanism. Moreover, the vast and accumulating preclinical data make it possible to design and carry out human studies to evaluate the efficacy of dietary ITC in cancer prevention and treatment, which may be facilitated by the use of plants and plant extracts that are rich in ITC.

\section{Acknowledgements}

We acknowledge helpful discussions with members of our laboratory, including Hillary E. Jobson, Joseph D. Paonessa and Li Tang. This work and the studies performed in authors' laboratory have been supported by National Cancer Institute grants CA 80962 and CA100623.

\section{References}

Ashkenazi A \& Dixit VM (1998) Death receptors: signaling and modulation. Science 281, 1305-1308.

Brusewitz G, Cameron BD, Chasseaud LF, Gorler K, Hawkins DR, Koch H \& Mennicke WH (1977) The metabolism of benzyl isothiocyanate and its cysteine conjugate. Biochemical Journal 162, 99-107.

Buttke TM \& Sandstrom PA (1994) Oxidative stress as a mediator of apoptosis. Immunology Today 15, 7-10.

Callaway EC, Zhang Y, Chew W \& Chow HHS (2004) Cellular accumulation of dietary anticarcinogenic isothiocyanates is followed by transporter-mediated export as dithiocarbamates. Cancer Letters 204, 23-31.

Chen YR, Wang W, Kong AN \& Tan TH (1998) Molecular mechanisms of c-Jun $\mathrm{N}$-terminal kinase-mediated apoptosis induced by anticarcinogenic isothiocyanates. Journal of Biological Chemistry 273, 1769-1775.

Chiao JW, Chung FL, Kancherla R, Ahmed T, Mittelman A \& Conaway CC (2002) Sulforaphane and its metabolite mediate growth arrest and apoptosis in human prostate cancer cells. International Journal of Oncology 20, 631-636.

Chiao JW, Chung FL, Krzeminski J, Amin S, Arshad R, Ahmed T \& Conaway CC (2000) Modulation of growth of human prostate cancer cells by the $\mathrm{N}$-acetylcysteine conjugate of phenethyl isothiocyanate. International Journal of Oncology 16, 1215-1219.

Chiao JW, Wu H, Ramaswamy G, Conaway CC, Chung FL, Wang L \& Liu D (2004) Ingestion of an isothiocyanate metabolite from cruciferous vegetables inhibits growth of human prostate cancer cell xenografts by apoptosis and cell cycle arrest. Carcinogenesis 25, 1403-1408.

Choi S \& Singh SV (2005) Bax and Bak are required for apoptosis induction by sulforaphane, a cruciferous vegetablederived cancer chemopreventive agent. Cancer Research 65, 2035-2043.

Conaway CC, Krzeminski J, Amin S \& Chung FL (2001) Decomposition rates of isothiocyanate conjugates determine their activity as inhibitors of cytochrome p450 enzymes. Chemical Research in Toxicology 14, 1170-1176.

Conaway CC, Yang YM \& Chung FL (2002) Isothiocyanates as cancer chemopreventive agents: their biological activities and metabolism in rodents and humans. Current Drug Metabolism 3, 233-255.

Fahey JW, Zhang Y \& Talalay P (1997) Broccoli sprouts: an exceptionally rich source of inducers of enzymes that protect against chemical carcinogens. Proceedings of the National Academy of Sciences USA 94, 10367-10372.

Fenwick GR, Heaney RK \& Mullin WJ (1983) Glucosinolates and their breakdown products in food and food plants. Critical Reviews in Food Science and Nutrition 18, 123-201.

Fowke JH, Chung FL, Jin F, Qi D, Cai Q, Conaway C, Cheng JR, Shu XO, Gao YT \& Zheng W (2003) Urinary isothiocyanate levels, Brassica, and human breast cancer. Cancer Research 63, 3980-3986.

Gamet-Payrastre L, Lumeau S, Gasc N, Cassar G, Rollin P \& Tulliez J (1998) Selective cytostatic and cytotoxic effects of glucosinolates hydrolysis products on human colon cancer cells in vitro. Anti-Cancer Drugs 9, 141-148.

Hall AG (1999) The role of glutathione in the regulation of apoptosis. European Journal of Clinical Investigation 29, $238-245$.

Hasegawa T, Nishino H \& Iwashima A (1993) Isothiocyanates inhibit cell cycle progression of $\mathrm{HeLa}$ cells at $\mathrm{G}_{2} / \mathrm{M}$ phase. Anti-Cancer Drugs 4, 273-279.

Hecht SS (2000) Inhibition of carcinogenesis by isothiocyanates. Drug Metabolism Reviews 32, 395-411.

Hu R, Kim BR, Chen C, Hebbar V \& Kong AN (2003) The role of JNK and apoptotic signaling pathways in PEITC-mediated responses in human HT-29 colon adenocarcinoma cells. Carcinogenesis 24, 1361-1367.

$\mathrm{Hu}$ K \& Morris ME (2004) Effects of benzyl-, phenethyl-, and alpha-naphthyl isothiocyanate on P-glycoprotein- and MRP1-mediated transport. Journal of Pharmaceutical Sciences 93, 1901-1911.

Huang C, Ma W, Li J, Hecht SS \& Dong Z (1998) Essential role of p53 in phenethyl isothiocyanate-induced apoptosis. Cancer Research 58, 4102-4106.

Hudson TS, Stoner GD, Morse MA, Young H \& Mallery SR (2005) Comparison of phenethyl and 6-phenylhexyl isothiocyanate-induced toxicity in rat esophageal cell lines with and without glutathione depletion. Toxicology Letter $\mathbf{1 5 5}$, 427-436.

Insinga A, Monestiroli S, Ronzoni S, Gelmetti V, Marchesi F, Viale A, Altucci L, Nervi C, Minucci S \& Pelicci PG (2005) Inhibitors of histone deacetylases induce tumor-selective apoptosis through activation of the death receptor pathway. Nature Medicine 11, 71-76.

Ioannou YM, Burka LT \& Matthews HB (1984) Allyl isothiocyanate: comparative disposition in rats and mice. Toxicology and Applied Pharmacology 75, 173-181.

Jackson SJ \& Singletary KW (2004a) Sulforaphane: a naturally occurring mammary carcinoma mitotic inhibitor, which disrupts tubulin polymerization. Carcinogenesis 25, 219-227.

Jackson SJT \& Singletary KW (2004b) Sulforaphane inhibits human MCF-7 mammary cancer cell mitotic progression and tubulin polymerization. Journal of Nutrition 134, 2229-2236.

Ji Y \& Morris ME (2003) Determination of phenethyl isothiocyanate in human plasma and urine by ammonia derivatization and liquid chromatography-tandem mass spectrometry. Analytical Biochemistry 323, 39-47.

Ji Y \& Morris ME (2004) Effect of organic isothiocyanates on breast cancer resistance protein (ABCG-2)-mediated transport. Pharmaceutical Research 21, 2261-2269. 
Kaum YS, Jeong WS \& Kong ANT (2004) Chemoprevention by isothiocyanates and their underlying molecular signaling mechanisms. Mutation Research 555, 191-202.

Kim BR, Hu R, Keum YS, Hebbar V, Shen G, Nair SS \& Kong ANT (2003) Effects of glutathione on antioxidant response element-mediated gene expression and apoptosis elicited by sulforaphane. Cancer Research 64, 7520-7525.

Lea MA, Randolph VM, Lee JE \& Des Bordes C (2001) Induction of histone acetylation in mouse erythroleukemia cells by some organosulfur compounds including allyl isothiocyanate. International Journal of Cancer 92, 784-789.

Li H, Zhu H, Xu CJ \& Yuan J (1998) Cleavage of BID by caspase- 8 mediates the mitochondrial damage in the Fas pathway of apoptosis. Cell 94, 491-501.

Li J, Yao S \& Zhang Y (2005) The role of c-Jun in the AP-1 activation induced by naturally occurring isothiocyanates. Food and Chemical Toxicology 43, 1373-1380.

London SJ, Yuan JM, Chung FL, Gao YT, Coetzee GA, Rose RK \& Yu MC (2000) Isothiocyanates, glutathione S-transferase M1 and T1 polymorphisms, and lung-cancer risk: A prospective study of men in Shanghai, China. Lancet 356, 724-729.

Lui VWY, Wentzel AL, Xiao D, Lew KL, Singh SV \& Grandis JR (2003) Requirement of a carbon spacer in benzyl isothiocyanate-mediated cytotoxicity and MAPK activation in head and neck squamous cell carcinoma. Carcinogenesis 24, $1705-1712$.

Miko M \& Chance B (1975) Isothiocyanates: a new class of uncouplers. Biochimica et Biophysica Acta 396, 165-174.

Miyashita T \& Reed JC (1995) Tumor suppressor p53 is a direct transcriptional activator of the human bax gene. Cell 80, 293-299.

Miyoshi N, Uchida K, Osawa T \& Nakamura Y (2004) A link between benzyl isothiocyanate-induced cell cycle arrest and apoptosis: involvement of mitogen-activated protein kinases in the Bcl-2 phosphorylation. Cancer Research 64, 2134-2142.

Musk SRR \& Johnson IT (1993) Allyl isothiocyanate is selectively toxic to transformed cells of the human colorectal tumour line HT29. Carcinogenesis 14, 2079-2083.

Myzak MC, Karplus PA, Chung FL \& Dashwood RH (2004) A novel mechanism of chemoprotection by sulforaphane: inhibition of histone deacetylase. Cancer Research 64, 5767-5774.

Nakamura Y, Kawakami M, Yoshihiro A, Miyoshi N, Ohigashi H, Kawai K, Osawa T \& Uchida K (2002) Involvement of the mitochondrial death pathway in chemopreventive benzyl isothiocyanate-induced apoptosis. Journal of Biological Chemistry 277, 8492-8499.

Nakamura Y, Ohigashi H, Masuda S, Murakami A, Morimitsu Y, Kawamoto Y, Osawa T, Imagawa M \& Uchida K (2000) Redox regulation of glutathione S-transferase induction by benzyl isothiocyanate: correlation of enzyme induction with the formation of reactive oxygen intermediates. Cancer Research 60, 219-225.

Nebbioso A, Clarke N, Voltz E, Germain E, Ambrosino C, Bontempo P et al. (2005) Tumor-selective action of HDAC inhibitors involves TRAIL induction in acute myeloid leukemia cells. Nature Medicine 11, 77-84.

Payen L, Courtois A, Loewert M, Guillouzo A \& Fardel Q (2001) Reactive oxygen species-related induction of multidrug resistance-associated protein 2 expression in primary hepatocytes exposed to sulforaphane. Biochemical and Biophysical Research Communications 282, 257-263.

Pham NA, Jacobberger JW, Schimmer AD, Cao P, Gronda M \& Hedley DW (2004) The dietary isothiocyanate sulforaphane targets pathways of apoptosis, cell cycle arrest, and oxidative stress in human pancreatic cancer cells and inhibits tumor growth in severe combined immunodeficient mice. Molecular Cancer Therapeutics 3, 1239-1248.

Rose P, Armstrong JS, Chua YL, Ong CN \& Whiteman M (2005) $\beta$-phenylethyl isothiocyanate mediated apoptosis; contribution of Bax and the mitochondrial death pathway. International Journal of Biochemistry and Cell Biology 37, $100-119$.

Rose P, Whiteman M, Huang SH, Halliwell B \& Ong CN (2003) $\beta$-phenylethyl isothiocyanate-mediated apoptosis in hepatoma HepG2 cells. Cellular and Molecular Life Sciences 60, 1489-1503.

Sasaki S (1963) Inhibitory effects by $\alpha$-naphthyl-isothiocyanate on development of hepatoma in rats treated with 3-methyl-4dimethyl-aminoazobenzene. Journal of Nara Medical Association 14, 101-115.

Scheid MP, Schubert KM \& Duronio V (1999) Regulation of Bad phosphorylation and association with $\mathrm{Bcl}-\mathrm{x}(\mathrm{L})$ by the MAPK/Erk kinase. Journal of Biological Chemistry 274, 31108-31113.

Seow A, Yuan JM, Sun CL, Van Den Berg D, Lee HP \& Yu MC (2002) Dietary isothiocyanates, glutathione S-transferase polymorphisms and colorectal cancer risk in the Singapore Chinese Health Study. Carcinogenesis 23, 2055-2061.

Shackelford RE, Kaufmann WK \& Paules RS (2000) Oxidative stress and cell cycle checkpoint function. Free Radical Biology and Medicine 28, 1387-1404.

Sidransky H, Ito N \& Verney E (1966) Influence of alphanaphthyl-isothiocyanate on liver tumorigenesis in rats ingesting ethionine and $\mathrm{N}$-2-fluorenylacetamide. Journal of the National Cancer Institute 37, 677-686.

Singh AV, Xiao D, Lew KL, Dhir R \& Singh SV (2004) Sulforaphane induces caspase-mediated apoptosis in cultured PC-3 human prostate cancer cells and retards growth of PC-3 xenografts in vivo. Carcinogenesis 25, 83-90.

Singh SV, Herman-Antosiewicz A, Singh AV, Lew KL, Srivastava SK, Kamath R, Brown KD, Zhang L \& Baskaran R (2004) Sulforaphane-induced G2/M phase cell cycle arrest involves checkpoint kinase 2-mediated phosphorylation of cell division cycle 25C. Journal of Biological Chemistry 279, 25813-25822.

Singh SV, Srivastava SK, Choi S, Lew KL, Antosiewicz J, Xiao D et al. (2005) Sulforaphane-induced cell death in human prostate cancer cells is initiated by reactive oxygen species. Journal of Biological Chemistry 280, 19911-19924.

Smith TK, Lund EK, Parker ML, Clarke RG \& Johnson IT (2004) Allyl isothiocyanate causes mitotic block, loss of cell adhesion and disrupted cytoskeletal structure in HT-29 cells. Carcinogenesis 25, 1409-1415.

Spitz MR, Duphorne CM, Detry MA, Pillow PC, Amos CI, Lei L, de Andrade M, Gu X, Hong WK \& Wu X (2000) Dietary intake of isothiocyanates: Evidence of a joint effect with glutathione S-transferase polymorphisms in lung cancer risk. Cancer Epidemiology, Biomarkers \& Prevention 9, $1017-1020$

Srivastava SK \& Singh SV (2004) Cell cycle arrest, apoptosis induction and inhibition of nuclear factor kappa B activation in anti-proliferative activity of benzyl isothiocyanate against human pancreatic cancer cells. Carcinogenesis 25, 1701-1709.

Srivastava SK, Xiao D, Lew KL, Hershberger P, Kokkinakis DM, Johnson CS, Trump DL \& Singh SV (2003) Allyl isothiocyanate, a constituent of cruciferous vegetables, inhibits growth of PC-3 human prostate cancer xenografts in vivo. Carcinogenesis 24, 1665-1670.

Tang L \& Zhang Y (2004) Dietary isothiocyanates inhibit the growth of human bladder carcinoma cells. Journal of Nutrition 134, 2004-2010. 
Tang L \& Zhang Y (2005) Mitochondria are the primary target in ITC-induced apoptosis in human bladder cancer UM-UC-3 cells. Molecular Cancer Therapeutics 4, 1250-1259.

Thornberry NA \& Lazebnik Y (1998) Caspases: enemies within. Science 281, 1312-1316.

Xiao D, Johnson CS, Trump DL \& Singh SV (2004) Proteasomemediated degradation of cell division cycle $25 \mathrm{C}$ and cyclindependent kinase 1 in phenethyl isothiocyanate-induced $\mathrm{G}_{2}-\mathrm{M}$-phase cycle arrest in PC-3 human prostate cancer cells. Molecular Cancer Therapeutics 3, 567-576.

Xiao D \& Singh SV (2002) Phenethyl isothiocyanate-induced apoptosis in P53-deficient PC-3 human prostate cancer cell line is mediated by extracellular signal-regulated kinases. Cancer Research 62, 3615-3619.

Xiao D, Srivastava SK, Lew KL, Zeng Y, Hershberger P, Johnson CS, Trump DL \& Singh SV (2003) Allyl isothiocyanate, a constituent of cruciferous vegetables, inhibits proliferation of human prostate cancer cells by causing $\mathrm{G}_{2} / \mathrm{M}$ arrest and inducing apoptosis. Carcinogenesis 24, 891-897.

Xiao D, Zeng Y, Choi S, Lew KL, Nelson JB \& Singh SV (2005) Caspase-dependent apoptosis induction by phenethyl isothiocyanate, a cruciferous vegetables-derived cancer chemopreventive agents, is mediated by Bak and Bax. Clinical Cancer Research 11, 2670-2679.

Xu K \& Thornalley PJ (2000) Studies on the mechanism of the inhibition of human leukemia cell growth by dietary isothiocyanates and their cysteine adducts in vitro. Biochemical Pharmacology 60, 221-231.

Xu K \& Thornalley PJ (2001a) Involvement of glutathione metabolism in the cytotoxicity of the phenethyl isothiocyanate and its cysteine conjugate to human leukemia cells in vitro. Biochemical Pharmacology 61, 165-177.

Xu K \& Thornalley PJ (2001b) Signal transduction activated by the cancer chemopreventive isothiocyanates: cleavage of BID protein, tyrosine phosphorylation and activation of JNK. British Journal of Cancer 84, 670-673.

Ye L, Dinkova-Kostova A, Wade KL, Zhang Y, Shapiro TA \& Talalay P (2002) Quantitative determination of dithiocarbamates in human plasma, serum, erythrocytes and urine: pharmacokinetics of broccoli sprout isothiocyanates in humans. Clinica Chimica Acta 316, 43-52.
Ye L \& Zhang Y (2001) Total intracellular accumulation levels of dietary isothiocyanates determine their activity in elevation of cellular glutathione and induction of phase 2 detoxification enzymes. Carcinogenesis 22, 1987-1992.

Zhang Y (2000) Role of glutathione in the accumulation of anticarcinogenic isothiocyanates and their glutathione conjugates by murine hepatoma cells. Carcinogenesis $\mathbf{2 1}$, $1175-1182$.

Zhang Y (2001) Molecular mechanism of rapid cellular accumulation of anticarcinogenic isothiocyanates. Carcinogenesis 22, 425-431.

Zhang Y (2004) Cancer-preventive isothiocyanates: measurement of human exposure and mechanism of action. Mutation Research 555, 173-190.

Zhang Y \& Callaway EC (2002) High cellular accumulation of sulphoraphane, a dietary anticarcinogen, is followed by rapid transporter-mediated export as a glutathione conjugate. Biochemical Journal 364, 301-307.

Zhang Y, Gonzalez V \& Xu MJ (2002) Expression and regulation of glutathione S-transferase P1-1 in cultured human epidermal cells. Journal of Dermatological Science 30, 205-214.

Zhang Y, Li J \& Tang L (2005) Cancer-preventive isothiocyanates: dichotomous modulators of oxidative stress. Free Radical Biology and Medicine 38, 70-77.

Zhang Y \& Talalay P (1994) Anticarcinogenic activities of organic isothiocyanates: chemistry and mechanism. Cancer Research 54, 1976S-1981S.

Zhang Y \& Talalay P (1998) Mechanism of differential potencies of isothiocyanates as inducers of anticarcinogenic phase 2 enzymes. Cancer Research 58, 4632-4639.

Zhang Y \& Tang L (2004) Dietary isothiocyanates inhibit the growth of human bladder carcinoma cells. Journal of Nutrition 134, 2004-2010.

Zhang Y, Tang L \& Gonzalez V (2003) Selected isothiocyanates rapidly induce growth inhibition of cancer cells. Molecular Cancer Therapeutics 2, 1045-1052.

Zhao B, Seow A, Lee EJ, Poh WT, The M, End P, Wang YT, Tan WC, Yu MC \& Lee HP (2001) Dietary isothiocyanates, glutathione S-transferase-M1, -T1 polymorphisms and lung cancer risk among Chinese women in Singapore. Cancer Epidemiology, Biomarkers \& Prevention 10, 1063-1067. 